\title{
Radial-velocity Variations of Late-type Variables
}

\author{
Stephenson L.S. Yang ${ }^{1}$ \\ Department of Physics and Astronomy, University of Victoria, Victoria, \\ $B C$, Canada
}

Gordon A.H. Walker ${ }^{1}$

Department of Physics and Astronomy, University of British Columbia, Vancouver, BC, Canada

Ana M. Larson ${ }^{1}$

Department of Astronomy, University of Washington, Seattle, WA, USA

\begin{abstract}
Low-amplitude, radial-velocity variations of bright late-type stars were monitored at the 1.2-m telescope of the Dominion Astrophysical Observatory (DAO) with the hydrogen fluoride (HF) absorption-cell technique. Many of the stars appear to be semi-regular red variables (SRb and $\mathrm{SRc}$ ) and irregular red variables $(\mathrm{Lb})$. The radial-velocity amplitudes range from a few tens of meters per second to a few kilometers per second while the timescales of the variations appear to range from a few tens of days to a few hundreds of days. These irregular-looking velocities are analysed for multiperiodicities. There are also variations in the chromospheric Ca II 8662 index for a few of the variables.
\end{abstract}

\section{Introduction}

In conventional radial-velocity techniques, wavelength calibration in stellar spectra is limited by collimation and guiding errors. These errors can be largely eliminated by imposing absorption lines of known wavelengths directly onto the stellar spectra. One set of suitable absorption lines belong to the R-branch of the (3-0) vibration-rotation band of hydrogen fluoride (HF). The technique (Campbell \& Walker 1979) involves placing an absorption cell filled with HF gas into the stellar beam. The monel absorption cell and its sapphire windows are maintained at $100^{\circ} \mathrm{C}$ in order to avoid polymerisation of the $\mathrm{HF}$ molecules. Connected to the cell is a reservoir of liquid $\mathrm{HF}$ maintained at $0^{\circ} \mathrm{C}$ to ensure a constant and stable vapor pressure. Meanwhile, a Reticon detector is utilised to provide the necessary high-signal-to-noise spectra.

\footnotetext{
${ }^{1}$ Visiting Astronomer, Dominion Astrophysical Observatory, operated by the National Research Council of Canada
} 


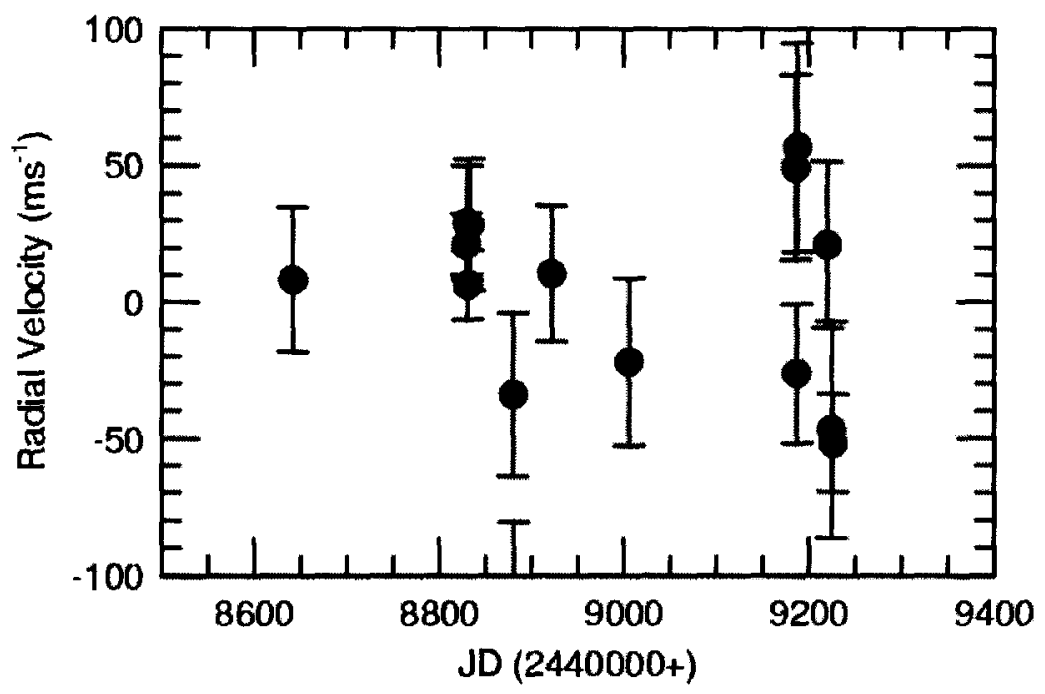

Figure 1. Scatter of $\alpha$ Ari velocities before application of run-to-run corrections.

\section{Observations}

The HF system is designed such that the cell can be easily moved in or out of the stellar beam. This enables the observations of spectra to be made with or without the imposed HF absorption lines. The spectrograph is centered at $\lambda 8700$ and has a reciprocal dispersion of $4.8 \AA / \mathrm{mm}$, giving a plate factor of about $0.071 \AA /$ pixel and a coverage of $130 \AA$ on the 1872 -pixel Reticon (Walker, Johnson, \& Yang 1985). In measuring a relative radial velocity for a spectrum, the effect of line blending between the stellar and HF lines can be minimised using numerical division by the reference stellar and/or HF spectra. The velocityreduction procedure has been described in Campbell et al. (1986).

The original HF system was used in a 12-year program at the CanadaFrance-Hawaii Telescope (CFHT) to monitor GK dwarfs for signatures of extrasolar planets (Campbell, Walker, \& Yang 1988; Walker et al. 1992, 1995). After the discovery that all the IAU radial-velocity standards used in the CFHT program are variable (Walker et al. 1989), a second HF cell was installed at the DAO $1.2-\mathrm{m}$ telescope to monitor the small-amplitude radial-velocity variations in bright, late-type stars (see also Larson, Yang, \& Walker 1999).

\section{Results}

The scatter in the velocities of $\alpha$ Ari (Figure 1) provides an indication of the external errors even before any run-to-run correction (of the order of $40 \mathrm{~m} / \mathrm{s}$ ) is 


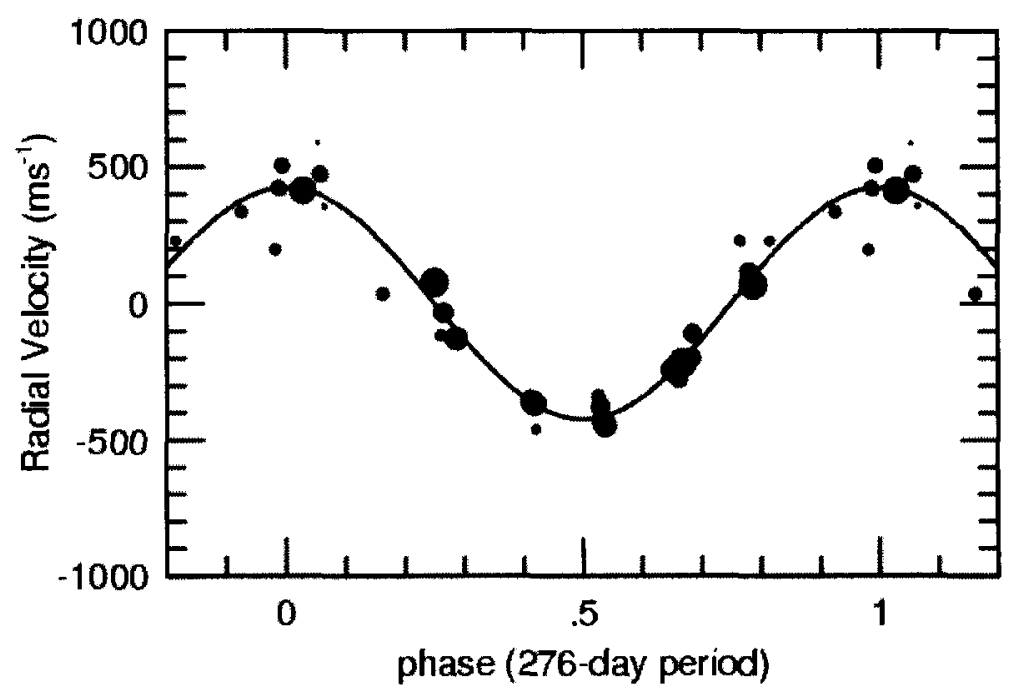

Figure 2. $\mu$ Gem velocities phased with one of three periods (after prewhitening the other two periods and phase-plotting only the one remaining period). The area of a point is inversely proportional to the square of its internal error.

applied. Due to slight fringing from one of the sapphire windows, these external errors are larger than the $13 \mathrm{~m} / \mathrm{s}$ achieved with the $\mathrm{HF}$ system at CFHT. The DAO HF obervations were made between 1991 and 1995. A telluric-line method has been used at DAO to measure radial velocities since 1995 (see Greimel \& Yang 1999).

Most of the observed M-type giants and supergiants appear to be variable. In fact, many of these stars are probably semiregular (SRb and SRc) and irregular (Lb) red variables. As an example, Figure 2 shows the velocities of $\mu \mathrm{Gem}$ phased with one in a set of three probable multiple periods. Generally, there are many almost-as-good-a-fit multiperiodic period-sets that would fit the observations for many of the program stars. Morever, these spectroscopic periods are generally different than known photometric periods (e.g. Percy \& Fleming 1992; Percy, Ralli, \& Sen 1993; Percy et al. 1994, 1996; Cristian et al. 1995). Many more additional observations will be needed to distinguish between the various multiperiodic solutions and uncover true periods. Of course, any irregular or chaotic variation would also complicate the problem.

The HF data also yield information on the simultaneous variability in the stellar temperature (Bohlender et al. 1992) and Ca II index (Larson et al. 1993). As an example, Figure 3 shows both the velocities and $\mathrm{Ca}$ II indices for $\mu \mathrm{Cep}$ phased to similar periods. 

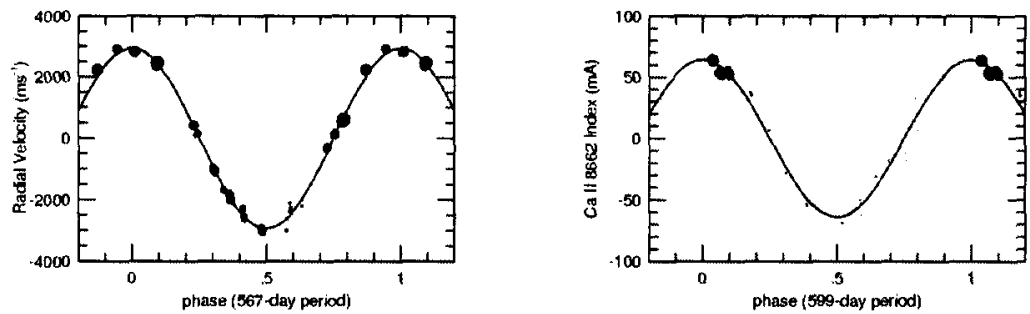

Figure 3. Phase plots of the $\mu$ Cep velocities and Ca II indices. Other periods have been prewhitened from the phased data points.

Acknowledgments. We are very grateful to Bruce Campbell for his pioneering work and ideas on the HF program. Alan Irwin has long supported this work with his ideas and the use of his periodogram programs. Moreover, Alan also upgraded and ported the data-reduction system onto unix. We would also like to thank Don VandenBerg for his support.

\section{References}

Bohlender, D.A., Irwin, A.W., Yang, S., \& Walker, G. A. H. 1992, PASP, 104, 1152

Campbell, B., \& Walker, G.A.H. 1979, PASP, 91, 540

Campbell, B., Walker, G.A.H., Pritchet, C., \& Long, B. 1986, in Astrophysics of Brown Dwarfs, M.C. Kafatos, R.S. Harrington, \& S. P. Maran, Cambridge: Cambridge University Press, 37

Campbell, B., Walker, G.A.H., \& Yang, S. 1988, ApJ, 331, 902

Cristian, V.C., Donahue, R.A., Soon, W.H., Baliunas, S.L., \& Henry, G.W. 1995, PASP, 107, 411

Greimel, R., \& Yang, S.L.S. 1999, these Proceedings

Larson, A.M., Irwin, A.W., Yang, S.L.S., Goodenough, C., Walker, G.A.H., Walker, A.R., \& Bohlender, D.A. 1993, PASP, 105, 332

Larson, A.M., Yang, S.L.S., \& Walker, G.A.H. 1999, these Proceedings

Percy, J.R., Desjardins, A., Yu, L., \& Landis, H. J. 1996, PASP, 108, 139

Percy, J.R., \& Fleming, D.E.B. 1992, PASP, 104, 96

Percy, J.R., Ralli, J.A., \& Sen, L.V. 1993, PASP, 101893

Percy, J.R., et al. (18 authors) 1994, PASP, 106, 611

Walker, G.A.H., Bohlender, D.A., Walker, A.R., Yang, A., \& Larson, A. 1992, ApJ, 396, L91

Walker, G.A.H., Johnson, R., \& Yang, S. 1985, Advances in Electronics and Electron Physics, 64A, 213

Walker, G.A.H., Walker, A.R., Irwin, A.W., Larson, A.M., Yang, S.L.S., \& Richardson, D.C. 1995, Icarus, 116359 
Walker, G.A.H., Yang, S., Campbell, B., \& Irwin, A.W. 1989, ApJ, 343, L21

\section{Discussion}

Queloz: What is your experience with the stability, from month to month, of telluric lines, which may depend on pressure, wind speed, etc.

Yang: The current program is still very much in progress, so we've not yet tested the stability of telluric lines. However, the effects of pressure-induced shifts of the $\mathrm{O}_{2}$ lines are known; see, for example, Campbell \& Walker (1979, PASP, 91, 540 ). The variability of the $\mathrm{H}_{2} \mathrm{O}$ lines is probably even more intense. In fact, different $\mathrm{H}_{2} \mathrm{O}$ lines will have different growth rates, making any compensation effort difficult. The effect of variable blending between stellar and telluric lines, especially with the weaker lines, may well be an ultimate limiting factor for the telluric-line technique; see Campbell (1983, PASP, 95, 577). Anyway, with the lower dispersion of only $1.0 \mathrm{~nm} / \mathrm{mm}$, I would be happy with a precision of 70 $\mathrm{m} / \mathrm{s}$.

W. Cochran: Have you experimented with reducing data with the $\mathrm{O}_{2}$ and $\mathrm{H}_{2} \mathrm{O}$ lines separately, and if so, which works better?

Yang: We haven't tried. But here I would expect the $\mathrm{H}_{2} \mathrm{O}$ line strength to change substantially quite often. I imagine $\mathrm{O}_{2}$ would be more stable.

Hummel: You presented results on 93 Leonis. Have you measured the A component too? The Mark III interferometer has measured the relative orbit.

Yang: We are engaged in a program, with Phil Bennett of Boulder, to observe 93 Leo near $380 \mathrm{~nm}$, in addition to the red region presented. The short-wavelength region will enable the measurement of the early-type component. 